Supporting Information

on

\title{
Diastereoselective Aldol Reactions of Enolates Generated from Vicinally Substituted Trimethylsilylmethyl Cyclopropyl Ketones
}

by

\author{
Veejendra K. Yadav* and Rengarajan Balamurugan \\ Department of Chemistry, Indian Institute of Technology, Kanpur 208 016, India \\ E-mail: vijendra@iitk.ac.in; FAX: Int. Code-91-512-2597436
}

General

${ }^{1} \mathrm{H}$ and ${ }^{13} \mathrm{C}$ spectra were recorded in $\mathrm{CDCl}_{3}$ solutions. The ${ }^{1} \mathrm{H}$ and ${ }^{13} \mathrm{C}$ spectra were referred, respectively, to TMS used as an internal standard and the central line for $\mathrm{CDCl}_{3}$. Elemental $(\mathrm{C}, \mathrm{H}, \mathrm{N})$ analyses were done on Perkin-Elmer 240-C automatic elemental analyzer. All the reactions were carried out using freshly distilled and dry solvents $\left(\mathrm{CH}_{2} \mathrm{Cl}_{2}\right.$ and benzene) from solvent stills. Column chromatography was performed over silica gel (100-200 mesh) from Acme Chemicals using hexanes and ethyl acetate mixtures as eluent. The separation of isomers and their rigorous purification were achieved by radial chromatography using plates coated with silica gel $\mathrm{PF}_{254}$ (E-Merck). Except few compounds all the compounds could be separated by radial chromatography. Solvents were removed under reduced pressure on a rotovap. Organic extracts were dried with anhydrous $\mathrm{Na}_{2} \mathrm{SO}_{4}$.

Typical procedure for the $\mathrm{TiCl}_{4}$-assisted reaction of the cyclopropane derivative $1 \mathrm{a}$ with benzaldehyde

A freshly prepared solution of $\mathrm{TiCl}_{4}(0.114 \mathrm{~g}, 0.75 \mathrm{mmol})$ in anhydrous $\mathrm{CH}_{2} \mathrm{Cl}_{2}(0.5 \mathrm{~mL})$ was added to a solution of $\mathbf{1 a}(0.116 \mathrm{~g}, 0.5 \mathrm{mmol})$ in anhydrous $\mathrm{CH}_{2} \mathrm{Cl}_{2}(2 \mathrm{~mL})$. The contents were stirred for $30 \mathrm{~min}$ that resulted in a red color solution. A solution of $\mathrm{PhCHO}(0.080 \mathrm{~g}$, $0.75 \mathrm{mmol})$ in anhydrous $\mathrm{CH}_{2} \mathrm{Cl}_{2}(0.5 \mathrm{~mL})$ was added. Decoloration commenced as the reaction proceeded. It was stirred for $1 \mathrm{~h}$ and then quenched with a solution of cold $2 \% \mathrm{HCl}$ $(5 \mathrm{~mL})$. Ether $(20 \mathrm{~mL})$ was added to it and the layers were separated. The organic layer was washed with cold $2 \% \mathrm{HCl}$ solution $(5 \mathrm{~mL})$. The combined aqueous layers were extracted with ether $(2 \times 5 \mathrm{~mL})$. The combined organic extracts were washed with water and brine, and dried. Removal of the solvents furnished the crude product that was purified by column chromatography (EtOAc/hexanes) to isolate 2-allyl-1,3-diphenyl-3-hydroxy-1-propanone, 0.1 g, 75\%, colorless viscous oil. Separation of the syn and anti isomers was achieved by radial chromatography. 


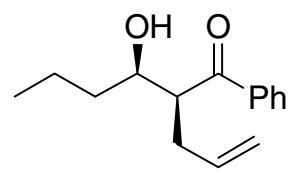

${ }^{1} \mathrm{H}$ NMR $\left(400 \mathrm{MHz}, \mathrm{CDCl}_{3}\right): \delta$ 7.95-7.93 $(2 \mathrm{H}, \mathrm{m}), 7.59-7.57(1 \mathrm{H}, \mathrm{m}), 7.50-7.46(2 \mathrm{H}, \mathrm{m})$, 5.81-5.71 $(1 \mathrm{H}, \mathrm{tdd}, J=17.1,10.0,7.1 \mathrm{~Hz}), 5.06-5.00(1 \mathrm{H}, \mathrm{qd}, J=16.8,1.5 \mathrm{~Hz}), 4.93(1 \mathrm{H}, \mathrm{d}$, $J=10.0 \mathrm{~Hz}), 3.98-3.94(1 \mathrm{H}, \mathrm{m}), 3.62-3.58(1 \mathrm{H}, \mathrm{m}), 2.65-2.52(2 \mathrm{H}, \mathrm{m}), 1.60-1.32(4 \mathrm{H}, \mathrm{m})$, $0.91(3 \mathrm{H}, \mathrm{t}, J=7.1 \mathrm{~Hz}) .{ }^{13} \mathrm{C}$ NMR $\left(100 \mathrm{MHz}, \mathrm{CDCl}_{3}\right): \delta 204.4,137.2,135.9,133.4,128.7$, 128.5, 116.8, 71.7, 50.6, 37.1, 31.8, 19.3, 13.9. Anal. Calcd. for $\mathrm{C}_{15} \mathrm{H}_{20} \mathrm{O}_{2}$ : C, 77.55; H, 8.68. Found C, 77.46; H, 8.55.

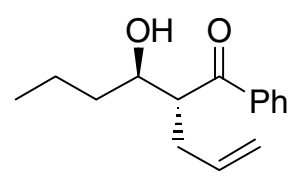

${ }^{1} \mathrm{H}$ NMR $\left(400 \mathrm{MHz}, \mathrm{CDCl}_{3}\right): \delta$ 7.95-7.93 $(2 \mathrm{H}, \mathrm{m}), 7.60-7.57(1 \mathrm{H}, \mathrm{m}), 7.51-7.58(2 \mathrm{H}, \mathrm{m})$, 5.81-5.71 (1H, tdd, $J=17.1,10.0,7.1 \mathrm{~Hz}), 5.11-5.05(1 \mathrm{H}, \mathrm{qd}, J=17.1,1.5 \mathrm{~Hz}), 5.00(1 \mathrm{H}, \mathrm{d}$, $J=10.0 \mathrm{~Hz}), 3.91-3.87(1 \mathrm{H}, \mathrm{m}), 3.61-3,56(1 \mathrm{H}, \mathrm{dt}, J=6.8,4.6 \mathrm{~Hz}), 2.54(2 \mathrm{H}, \mathrm{t}, J=7.1 \mathrm{~Hz})$, 1.60-1.32 (4H, m), $0.89(3 \mathrm{H}, \mathrm{t}, J=7.1 \mathrm{~Hz}) .{ }^{13} \mathrm{C}$ NMR $\left(100 \mathrm{MHz}, \mathrm{CDCl}_{3}\right): \delta 205.7,137.5$, 134.9, 133.5, 128.8, 128.3, 117.4, 72.3, 50.2, 37.9, 34.5, 19.2, 13.9. Anal. Calcd. for $\mathrm{C}_{15} \mathrm{H}_{20} \mathrm{O}_{2}: \mathrm{C}, 77.55 ; \mathrm{H}, 8.68$. Found $\mathrm{C}, 77.38 ; \mathrm{H}, 8.50$.

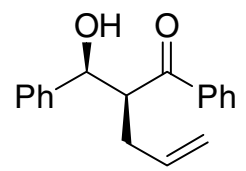

${ }^{1} \mathrm{H}$ NMR $\left(400 \mathrm{MHz}, \mathrm{CDCl}_{3}\right): \delta 7.84(2 \mathrm{H}, \mathrm{d}, J=7.3 \mathrm{~Hz}), 7.55-7.52(1 \mathrm{H}, \mathrm{m}), 7.43-7.37(4 \mathrm{H}$, m), 7.31-7.28 (2H, m), 7.23-7.19 $(1 \mathrm{H}, \mathrm{m}), 5.65-5.54(1 \mathrm{H}, \mathrm{tdd}, J=17.1,9.9,7.1 \mathrm{~Hz}), 5.10$ $(1 \mathrm{H}, \mathrm{d}, J=4.6 \mathrm{~Hz}), 4.94-4.89(1 \mathrm{H}, \mathrm{dd}, J=17.1,1.2 \mathrm{~Hz}), 4.86-4.83(1 \mathrm{H}, \mathrm{dd}, J=10.0,1.0 \mathrm{~Hz})$, 3.88-3.83 (1H, td, $J=8.9,4.5 \mathrm{~Hz}), 3.26(1 \mathrm{H}, \mathrm{s}), 2.68-2.61(1 \mathrm{H}, \mathrm{m}), 2.52-2.46(1 \mathrm{H}, \mathrm{m}) .{ }^{13} \mathrm{C}$ NMR (100 MHz, $\left.\mathrm{CDCl}_{3}\right): \delta 204.2,141.7,137.0,135.4,133.3,128.6,128.4,128.3,127.5$, 126.2, 116.9, 73.7, 52.7, 31.7. Anal. Calcd. for $\mathrm{C}_{18} \mathrm{H}_{18} \mathrm{O}_{2}$ : C, 81.17; H, 6.81. Found C, 81.08; H, 6.73 .<smiles>C=CCC(C(=O)Pc1ccccc1)C(O)c1ccccc1</smiles> 
${ }^{1} \mathrm{H}$ NMR (400 MHz, $\left.\mathrm{CDCl}_{3}\right): \delta .7 .89-7.86(2 \mathrm{H}, \mathrm{m}), 7.56-7.52(1 \mathrm{H}, \mathrm{m}), 7.45-7.31(6 \mathrm{H}, \mathrm{m})$, 7.27-7.23 (1H, m), 5.67-5.57 (1H, tdd, $J=17.1,10.0,7.1 \mathrm{~Hz}), 5.02(1 \mathrm{H}, \mathrm{t}, J=6.1 \mathrm{~Hz}), 4.96$ $(1 \mathrm{H}, \mathrm{d}, J=17.1 \mathrm{~Hz}), 4.93(1 \mathrm{H}, \mathrm{d}, J=10.0 \mathrm{~Hz}), 3.93-3.88(1 \mathrm{H}, \mathrm{m}), 3.18(1 \mathrm{H}, \mathrm{d}, J=5.6 \mathrm{~Hz})$, 2.47-2.40 (1H, m), 2.32-2.26 (1H, m). ${ }^{13} \mathrm{C}$ NMR (100 MHz, $\left.\mathrm{CDCl}_{3}\right): \delta 204.8,142.4,137.9$, 134.4, 133.2, 128.6, 128.5, 128.3, 127.9, 126.4, 117.6, 75.5, 52.6, 34.7. Anal. Calcd. for $\mathrm{C}_{18} \mathrm{H}_{18} \mathrm{O}_{2}$ : C, 81.17; H, 6.81. Found C, 81.04; H, 6.71.

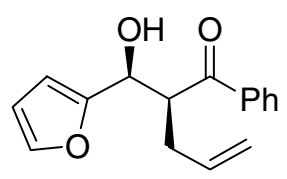

${ }^{1} \mathrm{H}$ NMR (400 MHz, $\left.\mathrm{CDCl}_{3}\right): \delta$ 7.91-7.89 (2H, m), 7.58-7.53 (1H, m), 7.46-7.42 (2H, m), $7.28(1 \mathrm{H}, \mathrm{t}, J=1.3 \mathrm{~Hz}), 6.25(2 \mathrm{H}, \mathrm{s}), 5.72-5.61(1 \mathrm{H}, \mathrm{tdd}, J=17.1,10.0,7.1 \mathrm{~Hz}), 5.10(1 \mathrm{H}, \mathrm{d}$, $J=5.4 \mathrm{~Hz}), 5.00-4.96(1 \mathrm{H}, \mathrm{dd}, J=17.1,1.6 \mathrm{~Hz}), 4.92-4.89(1 \mathrm{H}, \mathrm{dd}, J=10.0,0.9 \mathrm{~Hz}), 4.10-$ $4.05(1 \mathrm{H}, \mathrm{m}), 3.09\left(1 \mathrm{H}\right.$, broad s), 2.69-2.56 $(2 \mathrm{H}, \mathrm{m}) .{ }^{13} \mathrm{C} \mathrm{NMR}\left(100 \mathrm{MHz}, \mathrm{CDCl}_{3}\right): \delta 203.0$, 154.0, 141.7, 136.7, 135.0, 134.0, 133.4, 128.6, 128.4, 117.1, 110.3, 107.4, 107.1, 68.4, 50.2, 32.4. Anal. Calcd. for $\mathrm{C}_{16} \mathrm{H}_{16} \mathrm{O}_{3}$ : C, 74.98; H, 6.29. Found C, 74.90; H, 6.13.

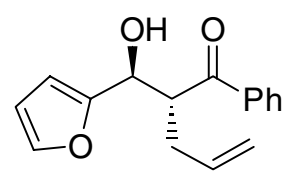

${ }^{1} \mathrm{H}$ NMR (400 MHz, $\left.\mathrm{CDCl}_{3}\right): \delta$ 7.95-7.90 (2H, m), 7.60-7.55 (1H, m), 7.49-7.44 (2H, m), 7.33-7.32 (1H, dd, $J=1.7,1.0 \mathrm{~Hz}), 6.29-6.27(2 \mathrm{H}, \mathrm{m}), 5.67-5.65(1 \mathrm{H}, \mathrm{tdd}, J=17.2,10.0,7.2$ $\mathrm{Hz}), 5.04(1 \mathrm{H}, \mathrm{t}, J=6.3 \mathrm{~Hz}), 5.04-4.96(2 \mathrm{H}, \mathrm{m}), 4.12-4.07(1 \mathrm{H}, \mathrm{q}, J=6.7 \mathrm{~Hz}), 3.43(1 \mathrm{H}, \mathrm{d}, J$ $=7.3 \mathrm{~Hz}), 2.45-2.40(2 \mathrm{H}, \mathrm{m}) .{ }^{13} \mathrm{C}$ NMR $\left(100 \mathrm{MHz}, \mathrm{CDCl}_{3}\right): \delta 204.4,154.8,142.0,137.2$, 134.0, 133.4, 128.7, 128.4, 117.8, 110.3, 107.4, 68.9, 49.4, 34.3. Anal. Calcd. for $\mathrm{C}_{16} \mathrm{H}_{16} \mathrm{O}_{3}$ : C, 74.98; H, 6.29. Found C, 74.87; H, 6.16.<smiles>C=CCC(C(=O)c1ccccc1)[C@H](O)c1ccc(OC)cc1Br</smiles>

${ }^{1} \mathrm{H}$ NMR (400 MHz, $\left.\mathrm{CDCl}_{3}\right): \delta$ 8.03-8.01 $(2 \mathrm{H}, \mathrm{m}), 7.62-7.58(2 \mathrm{H}, \mathrm{m}), 7.51-7.47(2 \mathrm{H}, \mathrm{m})$, 7.11- $7.10(1 \mathrm{H}, \mathrm{m}), 6.92-6.89(1 \mathrm{H}, \mathrm{m}), 5.55-5.44(1 \mathrm{H}, \mathrm{m}), 5.32(1 \mathrm{H}, \mathrm{s}), 4.88-4.83(1 \mathrm{H}, \mathrm{dd}, J$ $=16.9,1.5 \mathrm{~Hz}), 4.79(1 \mathrm{H}, \mathrm{d}, J=10.2 \mathrm{~Hz}), 4.08-4.03(1 \mathrm{H}, \mathrm{m}), 3.95(1 \mathrm{H}$, broad s $), 3.80(3 \mathrm{H}$, 
s), 2.62-2.54 (1H, m), 2.32-2.56 (1H, m). $\left.{ }^{13} \mathrm{C} \mathrm{NMR} \mathrm{(100} \mathrm{MHz,} \mathrm{CDCl}_{3}\right): \delta 205.8,159.4$, 136.8, 135.3, 133.7, 131.7, 129.5, 128.7, 121.5, 118.0, 116.9, 113.3, 71.7, 55.5, 48.4, 30.4 . Anal. Calcd. for $\mathrm{C}_{19} \mathrm{H}_{19} \mathrm{BrO}_{3}$ : C, 60.81; H, 5.10. Found C, 60.85; H, 5.01.

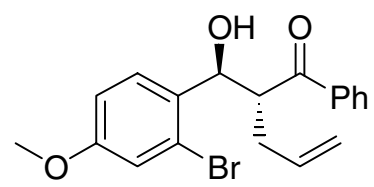

${ }^{1} \mathrm{H}$ NMR (400 MHz, $\left.\mathrm{CDCl}_{3}\right): \delta 7.95(2 \mathrm{H}, \mathrm{d}, J=7.3 \mathrm{~Hz}), 7.60-7.57(1 \mathrm{H}, \mathrm{m}), 7.51-7.45(2 \mathrm{H}$, m), $7.36(1 \mathrm{H}, \mathrm{d}, J=8.1 \mathrm{~Hz}), 7.12-7.10(1 \mathrm{H}, \mathrm{m}), 6.98(1 \mathrm{H}, \mathrm{d}, J=1.7 \mathrm{~Hz}), 5.60-5.50(1 \mathrm{H}, \mathrm{tdd}$, $J=17.1,10.2,7.1 \mathrm{~Hz}), 5.20(1 \mathrm{H}, \mathrm{t}, J=3.3 \mathrm{~Hz}), 4.90-4.86(1 \mathrm{H}, \mathrm{dd}, J=17.1,1.5 \mathrm{~Hz}), 4.83-$ $4.80(1 \mathrm{H}, \mathrm{dd}, J=10.2,1.0 \mathrm{~Hz}), 4.05-4.01(1 \mathrm{H}, \mathrm{td}, J=9.6,3.8 \mathrm{~Hz}), 3.87(3 \mathrm{H}, \mathrm{s}), 3.45(1 \mathrm{H}, \mathrm{d}$, $J=3.7 \mathrm{~Hz}), 2.67-2.58(1 \mathrm{H}, \mathrm{m}), 2.34-2.27(1 \mathrm{H}, \mathrm{m}) .{ }^{13} \mathrm{C} \mathrm{NMR}\left(100 \mathrm{MHz}, \mathrm{CDCl}_{3}\right): \delta 204.6$, 156.3, 137.0, 135.7, 133.4, 129.2, 128.8, 128.6, 128.5, 123.8, 121.7, 116.6, 113.9, 69.6, 55.6, 49.4, 30.7. Anal. Calcd. for $\mathrm{C}_{19} \mathrm{H}_{19} \mathrm{BrO}_{3}$ : C, 60.81; H, 5.10. Found C, 60.73; H, 4.97.<smiles>C=CCC(C(=O)c1ccccc1)C(O)/C=C/c1ccccc1</smiles>

Characteristic ${ }^{1} \mathrm{H}$ signals for the syn isomer: $\delta 6.69-6.65(1 \mathrm{H}, \mathrm{dd}, J=15.9,0.7 \mathrm{~Hz}), 6.26-6.21$ $(1 \mathrm{H}, \mathrm{dd}, J=15.9,6.4 \mathrm{~Hz}), 5.04-5.00(1 \mathrm{H}, \mathrm{dd}, J=17.1,1.5 \mathrm{~Hz}), 4.96-4.93(1 \mathrm{H}, \mathrm{dd}, J=10.0$, $1.0 \mathrm{~Hz}), 4.69(1 \mathrm{H}, \mathrm{t}, J=5.0 \mathrm{~Hz}), 2.91(1 \mathrm{H}, \mathrm{s})$

Characteristic ${ }^{1} \mathrm{H}$ signals for the anti isomer: $\delta 6.65(1 \mathrm{H}, \mathrm{d}, J=15.6 \mathrm{~Hz}), 6.26-6.20(1 \mathrm{H}, \mathrm{dd}, J$ $=15.9,6.8 \mathrm{~Hz}), 5.09-5.04(1 \mathrm{H}, \mathrm{dd}, J=16.8,1.2 \mathrm{~Hz}), 5.01-4.98(1 \mathrm{H}, \mathrm{dd}, J=10.0,1.0 \mathrm{~Hz})$, 4.64-4.60 (1H, broad m), $3.03(1 \mathrm{H}$, broad d, $J=4.8 \mathrm{~Hz})$.

${ }^{13} \mathrm{C}$ NMR $\left(100 \mathrm{MHz}, \mathrm{CDCl}_{3}\right)$ of the mixture: $\delta 204.5,203.8,137.5,137.2,136.5,136.4$, $135.5,134.6,133.4,134.9,131.4,130.0,129.1,128.7,128.5,128.4,127.8,127.7,126.5$, $117.6,117.1,73.8,72.8,51.1,51.0,34.3,32.1$.

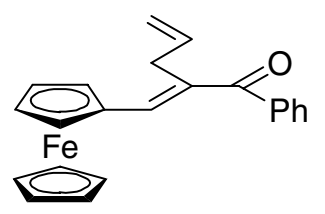


${ }^{1} \mathrm{H}$ NMR (400 MHz, $\left.\mathrm{CDCl}_{3}\right): \delta$ 7.72-7.69 $(2 \mathrm{H}, \mathrm{m}), 7.56-7.52(1 \mathrm{H}, \mathrm{m}), 7.49-7.45(2 \mathrm{H}, \mathrm{m})$, $7.08(1 \mathrm{H}, \mathrm{s}), 6.07-5.97(1 \mathrm{H}, \mathrm{m}), 5.22-5.17(1 \mathrm{H}, \mathrm{dd}, J=17.3,1.7 \mathrm{~Hz}), 5.25-5.12(1 \mathrm{H}, \mathrm{dd}, J=$ $10.0,1.7 \mathrm{~Hz}), 4.50-4.49(2 \mathrm{H}, \mathrm{t}, J=1.8 \mathrm{~Hz}), 4.43-4.42(2 \mathrm{H}, \mathrm{t}, J=1.8 \mathrm{~Hz}), 4.16(5 \mathrm{H}, \mathrm{s}), 3.45-$ $3.43(2 \mathrm{H}, \mathrm{td}, J=5.6,1.7 \mathrm{~Hz}) .{ }^{13} \mathrm{C}$ NMR $\left(100 \mathrm{MHz}, \mathrm{CDCl}_{3}\right): \delta 198.0,145.1,139.5,135.3$, 134.5, 131.4, 129.2, 128.1, 115.3, 78.5, 70.9, 70.7, 69.4, 31.5. Anal. Calcd. for $\mathrm{C}_{22} \mathrm{H}_{20} \mathrm{FeO}: \mathrm{C}$, 74.17; H, 5.66. Found C, 74.10; H, 5.55.

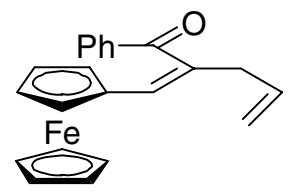

${ }^{1} \mathrm{H}$ NMR (400 MHz, $\left.\mathrm{CDCl}_{3}\right): \delta$ 7.91-7.89 (2H, m), 7.50-7.44 (1H, m), 7.41-7.37 (2H, m), $6.42(1 \mathrm{H}, \mathrm{s}), 5.93-5.85(1 \mathrm{H}, \mathrm{m}), 5.15-5.08(2 \mathrm{H}, \mathrm{m}), 4.08(5 \mathrm{H}, \mathrm{d}, J=1.0 \mathrm{~Hz}), 4.03(4 \mathrm{H}, \mathrm{s})$, 3.11-3.09 (2H, dd, $\left.J=6.8,1.0 \mathrm{~Hz}) .{ }^{13} \mathrm{C} \mathrm{NMR} \mathrm{(100} \mathrm{MHz,} \mathrm{CDCl}_{3}\right): \delta 200.4,137.3,136.1$, 134.7, 133.2, 129.4, 128.5, 128.0, 117.3, 80.3, 69.11, 69.08, 68.9, 40.1. Anal. Calcd. for $\mathrm{C}_{22} \mathrm{H}_{20} \mathrm{FeO}: \mathrm{C}, 74.17 ; \mathrm{H}$, 5.66. Found C, 74.04; H, 5.52.

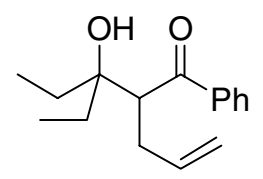

${ }^{1} \mathrm{H}$ NMR (400 MHz, $\left.\mathrm{CDCl}_{3}\right): \delta 7.93(2 \mathrm{H}, \mathrm{d}, J=7.6 \mathrm{~Hz}), 7.62-7.58(1 \mathrm{H}, \mathrm{m}), 7.50-7.47(2 \mathrm{H}$, m), 5.70-5.60 (1H, tdd, $J=17.1,10.0,7.3 \mathrm{~Hz}), 5.04-5.00(1 \mathrm{H}, \mathrm{dd}, J=17.1,1.4 \mathrm{~Hz}), 4.89$ $4.86(1 \mathrm{H}, \mathrm{dd}, J=10.0,1.0 \mathrm{~Hz}), 3.74-3.70(1 \mathrm{H}, \mathrm{dd}, J=10.1,4.3 \mathrm{~Hz}), 3.67(1 \mathrm{H}, \mathrm{s}), 2.67-2.60$ $(1 \mathrm{H}, \mathrm{m}), 2.56-2.50(1 \mathrm{H}, \mathrm{m}), 1.76-1.29(4 \mathrm{H}, \mathrm{m}), 0.90(3 \mathrm{H}, \mathrm{t}, J=7.4 \mathrm{~Hz}), 0.80(3 \mathrm{H}, \mathrm{t}, J=7.4$ $\mathrm{Hz}) .{ }^{13} \mathrm{C} \mathrm{NMR}\left(100 \mathrm{MHz}, \mathrm{CDCl}_{3}\right): \delta 208.1,138.6,135.7,133.6,128.7,128.4,117.1,76.6$, 49.5, 32.8, 30.4, 26.8, 7.9, 7.7. Anal. Calcd. for $\mathrm{C}_{16} \mathrm{H}_{22} \mathrm{O}_{2}$ : C, 78.01; H, 9.00. Found C, 77.95; $\mathrm{H}, 8.88$.

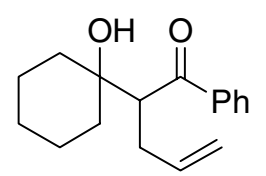

${ }^{1} \mathrm{H}$ NMR $\left(400 \mathrm{MHz}, \mathrm{CDCl}_{3}\right): \delta$ 7.95-7.93 $(2 \mathrm{H}, \mathrm{m}), 7.60-7.56(1 \mathrm{H}, \mathrm{m}), 7.49-7.46(2 \mathrm{H}, \mathrm{m})$, 5.71-5.61 (1H, tdd, $J=16.9,10.0,7.2 \mathrm{~Hz}), 5.04-4.98(1 \mathrm{H}, \mathrm{qd}, J=16.8,1.6 \mathrm{~Hz}), 4.89-4.86$ $(1 \mathrm{H}, \mathrm{td}, J=10.0,1.0 \mathrm{~Hz}), 3.65-3.62(1 \mathrm{H}, \mathrm{dd}, J=9.0,5.4 \mathrm{~Hz}), 3.44(1 \mathrm{H}, \mathrm{s}), 2.66-2.56(2 \mathrm{H}$, 
m), 1.89-1.16 (10 H, m). ${ }^{13} \mathrm{C}$ NMR (100 MHz, $\left.\mathrm{CDCl}_{3}\right): \delta 207.6,138.6,135.9,133.5,128.7$, 128.4, 117.0, 73.1, 53.2, 37.6, 35.3, 32.4, 25.7, 21.8, 21.7. Anal. Calcd. for $\mathrm{C}_{17} \mathrm{H}_{22} \mathrm{O}_{2}$ : C, 79.03; H, 8.58. Found C, 78.92; H, 8.40.

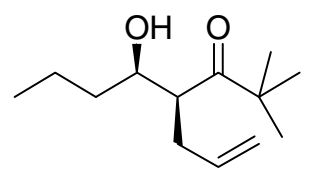

${ }^{1} \mathrm{H}$ NMR (400 MHz, $\left.\mathrm{CDCl}_{3}\right): \delta$ 5.72-5.62 (1H, tdd, $\left.J=17.1,10.0,7.3 \mathrm{~Hz}\right), 5.01-4.97(1 \mathrm{H}, \mathrm{dd}$, $J=17.1,1.5 \mathrm{~Hz}), 4.94-4.91(1 \mathrm{H}, \mathrm{dd}, J=10.0,1.0 \mathrm{~Hz}), 3.68-3.65(1 \mathrm{H}, \mathrm{m}), 3.09-3.04(1 \mathrm{H}, \mathrm{m})$, $2.85(1 \mathrm{H}, \mathrm{s}), 2.41-2.27(2 \mathrm{H}, \mathrm{m}), 1.56-1.40(2 \mathrm{H}, \mathrm{m}), 1.35-1.25(2 \mathrm{H}, \mathrm{m}), 1.09(9 \mathrm{H}, \mathrm{s}), 0.87(3 \mathrm{H}$, $\mathrm{t}, J=7.1 \mathrm{~Hz}) .{ }^{13} \mathrm{C}$ NMR $\left(100 \mathrm{MHz}, \mathrm{CDCl}_{3}\right): \delta 220.3,136.4,116.8,71.2,49.3,44.7,36.7$, 30.9, 26.1, 19.3, 13.9. Anal. Calcd. for $\mathrm{C}_{13} \mathrm{H}_{24} \mathrm{O}_{2}$ : C, 73.54; H, 11.39. Found C, 73.46; H, 11.29.<smiles>CC1(C)C=CC[C@H]([C@H](O)c2ccccc2)C1=O</smiles>

${ }^{1} \mathrm{H}$ NMR $\left(400 \mathrm{MHz}, \mathrm{CDCl}_{3}\right): \delta$ 7.35-7.23 $(5 \mathrm{H}, \mathrm{m}), 5.65-5.54(1 \mathrm{H}, \mathrm{m}), 4.97-4.89(3 \mathrm{H}, \mathrm{m})$, 3.39-3.35 (1H, td, $J=8.7,4.4 \mathrm{~Hz}), 3.22(1 \mathrm{H}$, broad s), 2.51-2.43 $(1 \mathrm{H}, \mathrm{m}), 2.30-2.24(1 \mathrm{H}, \mathrm{m})$, $1.02(9 \mathrm{H}, \mathrm{s}) .{ }^{13} \mathrm{C} \mathrm{NMR}\left(100 \mathrm{MHz}, \mathrm{CDCl}_{3}\right): \delta 219.4,141.7,136.0,127.2,127.5,126.2,116.9$, 73.6, 52.3, 44.8, 31.8, 26.0. Anal. Calcd. for $\mathrm{C}_{16} \mathrm{H}_{22} \mathrm{O}_{2}$ : C, 78.01; H, 9.00. Found C, 77.90; H, 8.86 .

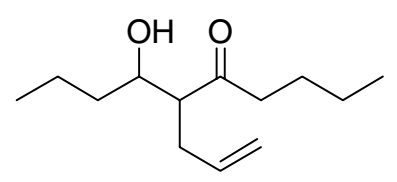

Characteristic ${ }^{1} \mathrm{H}$ signals for the syn isomer: $\delta 3.85-3.82(1 \mathrm{H}, \mathrm{m}), 0.92(3 \mathrm{H}, \mathrm{t}, J=7.1 \mathrm{~Hz})$.

Characteristic ${ }^{1} \mathrm{H}$ signals for the anti isomer: $\delta 3.74-3.71(1 \mathrm{H}, \mathrm{m}), 0.95(3 \mathrm{H}, \mathrm{t}, J=7.3 \mathrm{~Hz})$.

${ }^{13} \mathrm{C}$ NMR $\left(100 \mathrm{MHz}, \mathrm{CDCl}_{3}\right)$ of the mixture: $\delta 216.2,215.1,136.0,135.0,117.3,116.9,72.1$, 71.1, 56.1, 56.0, 44.8, 44.2, 37.7, 36.6, 33.8, 31.1, 25.1, 25.0, 22.2, 19.2, 19.0, 13.9, 13.8 . 
<smiles>C=CCC(C(=O)CCCC)C(O)c1ccccc1</smiles>

Characteristic ${ }^{1} \mathrm{H}$ signals for the syn isomer: $\delta 4.88(1 \mathrm{H}, \mathrm{d}, J=5.8 \mathrm{~Hz}), 3.03(1 \mathrm{H}, \mathrm{s}), 0.82$ $(3 \mathrm{H}, \mathrm{t}, J=7.3 \mathrm{~Hz})$.

Characteristic ${ }^{1} \mathrm{H}$ signals for the anti isomer: $\delta 4.80(1 \mathrm{H}, \mathrm{t}, J=6.2 \mathrm{~Hz}), 3.15(1 \mathrm{H}, \mathrm{d}, J=5.6$ $\mathrm{Hz}), 0.86(3 \mathrm{H}, \mathrm{t}, J=7.3 \mathrm{~Hz})$.

${ }^{13} \mathrm{C}$ NMR (100 MHz, $\mathrm{CDCl}_{3}$ ) of the mixture: $\delta$ 215.4, 214.4, 142.3, 141.8, 135.5, 134.5, $128.3,127.7,126.2,117.5,117.0,75.2,73.8,58.6,57.9,45.2,44.7,34.0,31.9,24.8,22.0$, 13.8, 13.7 .

Conversion of syn-2-allyl-1-phenyl-3-hydroxy-1-hexanone into tetrahydrofuranylmethanol derivatives

To a stirred solution of syn-2-allyl-1-phenyl-3-hydroxy-1-hexanone (0.05 g, 0.22 $\mathrm{mmol})$ in anhydrous benzene $(2 \mathrm{~mL}), \mathrm{m}$-CPBA $(0.095 \mathrm{~g}, 0.33 \mathrm{mmol}, 60 \%)$ was added in portions at $0-5^{\circ} \mathrm{C}$. The reaction mixture was stirred at the same temperature for $3 \mathrm{~h}$ and then warmed to room temperature. After stirring for $20 \mathrm{~h}$ at room temperature, the reaction mixture was quenched with saturated aqueous $\mathrm{Na}_{2} \mathrm{SO}_{3}$ solution $(5 \mathrm{~mL}) . \mathrm{Et}_{2} \mathrm{O}(15 \mathrm{~mL})$ was added and the layers were separated. The organic solution was washed with saturated aq $\mathrm{NaHCO}_{3}(2 \times 5 \mathrm{~mL})$. The combined aqueous washings were extracted with $\mathrm{Et}_{2} \mathrm{O}(2 \times 5 \mathrm{~mL})$ and the combined organic extracts were washed with brine, dried, and concentrated to obtain the crude product. This was purified by silica gel column chromatography (EtOAc/hexanes) to furnish a mixture of the corresponding tetrahydrofuranylmethanol derivatives, $0.038 \mathrm{~g}$, $71 \%$. Separation of the diastereomers was achieved by radial chromatography.

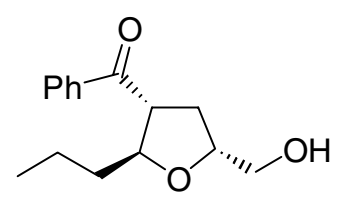

${ }^{1} \mathrm{H}$ NMR $\left(400 \mathrm{MHz}, \mathrm{CDCl}_{3}\right): \delta$ 7.97-7.95 $(2 \mathrm{H}, \mathrm{m}), 7.61-7.58(1 \mathrm{H}, \mathrm{m}), 7.51-7.47(2 \mathrm{H}, \mathrm{m})$, 4.39-4.34 (1H, m), 4.32-4.26 (1H, dq, $J=7.6,3.4 \mathrm{~Hz}), 3.79-3.73(1 \mathrm{H}, \mathrm{dt}, J=8.6,6.8 \mathrm{~Hz})$, 3.71-3.67 $(1 \mathrm{H}, \mathrm{dd}, J=11.7,3.3 \mathrm{~Hz}), 3.66-3.61(1 \mathrm{H}, \mathrm{dd}, J=11.7,6.1 \mathrm{~Hz}), 2.41-2.34(1 \mathrm{H}$, ddd, $J=12.4,8.6,7.1 \mathrm{~Hz}), 2.08-2.00(1 \mathrm{H}, \mathrm{td}, J=12.7,8.2 \mathrm{~Hz}), 1.68-1.58(1 \mathrm{H}, \mathrm{m}), 1.56-1.42$ $(2 \mathrm{H}, \mathrm{m}), 1.36-1.28(1 \mathrm{H}, \mathrm{m}), 0.90(3 \mathrm{H}, \mathrm{t}, J=7.1 \mathrm{~Hz}) .{ }^{13} \mathrm{C} \mathrm{NMR}\left(100 \mathrm{MHz}, \mathrm{CDCl}_{3}\right): \delta 199.9$, 
136.6, 133.4, 128.8, 128.4, 81.2, 78.9, 64.6, 51.9, 37.0, 33.0, 29.7, 19.4, 14.0. Anal. Calcd. for $\mathrm{C}_{15} \mathrm{H}_{20} \mathrm{O}_{3}$ : C, 72.55; H, 8.12. Found C, 72.38; H, 7.98.

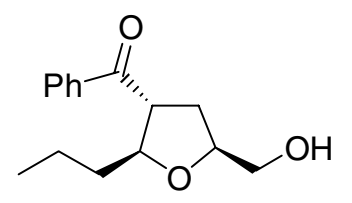

${ }^{1} \mathrm{H}$ NMR (400 MHz, $\left.\mathrm{CDCl}_{3}\right): \delta$ 7.97-7.95 $(2 \mathrm{H}, \mathrm{m}), 7.60-7.57(1 \mathrm{H}, \mathrm{m}), 7.51-7.47(2 \mathrm{H}, \mathrm{m})$, 4.32-4.27 (1H, dt, $J=7.6,4.6 \mathrm{~Hz}), 4.22-4.16(1 \mathrm{H}, \mathrm{m}), 3.83-3.80(1 \mathrm{H}, \mathrm{dd}, J=11.7,3.0 \mathrm{~Hz})$, 3.69-3.63 (1H, dt, $J=9.8,7.3 \mathrm{~Hz}), 3.57-3.53(1 \mathrm{H}, \mathrm{dd}, J=11.8,4.5 \mathrm{~Hz}), 2.27-2.20(1 \mathrm{H}$, ddd, $J=12.4,10.0,7.3 \mathrm{~Hz}), 2.19-2.12(1 \mathrm{H}, \mathrm{td}, J=12.4,7.3 \mathrm{~Hz}), 1.68-1.30(4 \mathrm{H}, \mathrm{m}), 0.90(3 \mathrm{H}, \mathrm{t}, J$ $=7.13 \mathrm{~Hz}) .{ }^{13} \mathrm{C} \mathrm{NMR}\left(100 \mathrm{MHz}, \mathrm{CDCl}_{3}\right): \delta 200.4,136.7,133.4,128.8,128.4,82.1,78.8$, 64.3, 51.6, 37.2, 33.1, 19.4, 14.1. Anal. Calcd. for $\mathrm{C}_{15} \mathrm{H}_{20} \mathrm{O}_{3}: \mathrm{C}, 72.55 ; \mathrm{H}, 8.12$. Found $\mathrm{C}$, $72.44 ; \mathrm{H}, 8.03$.

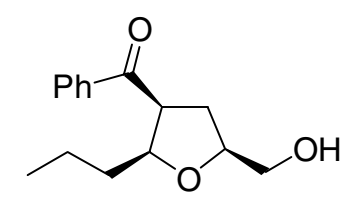

${ }^{1} \mathrm{H}$ NMR (400 MHz, $\left.\mathrm{CDCl}_{3}\right): \delta$ 7.97-7.95 $(2 \mathrm{H}, \mathrm{m}), 7.62-7.58(1 \mathrm{H}, \mathrm{m}), 7.51-7.48(2 \mathrm{H}, \mathrm{m})$, 4.30-4.25 (1H, m), 4.23-4.17 (1H, q, $J=7.6 \mathrm{~Hz}), 4.21-4.14(1 \mathrm{H}, \mathrm{m}), 3.92-3.89(1 \mathrm{H}, \mathrm{dd}, J=$ 11.6, $6.6 \mathrm{~Hz}), 3.72-3.67(1 \mathrm{H}, \mathrm{dd}, J=11.7,4.9 \mathrm{~Hz}), 2.65(1 \mathrm{H}$, broad s), 2.41-2.34 (1H, ddd, $J$ $=13.0,8.8,7.1 \mathrm{~Hz}), 2.15-2.08(1 \mathrm{H}, \mathrm{td}, J=13.0,7.3 \mathrm{~Hz}), 1.49-1.07(4 \mathrm{H}, \mathrm{m}), 0.77(3 \mathrm{H}, \mathrm{t}, J=$ $7.3 \mathrm{~Hz}) .{ }^{13} \mathrm{C}$ NMR $\left(100 \mathrm{MHz}, \mathrm{CDCl}_{3}\right): \delta 200.2,137.5,133.5,128.9,128.3,81.1,79.2,64.8$, 49.1, 34.2, 30.1, 19.8, 13.8. Anal. Calcd. for $\mathrm{C}_{15} \mathrm{H}_{20} \mathrm{O}_{3}: \mathrm{C}, 72.55 ; \mathrm{H}, 8.12$. Found $\mathrm{C}, 72.59 ; \mathrm{H}$, 8.06 .

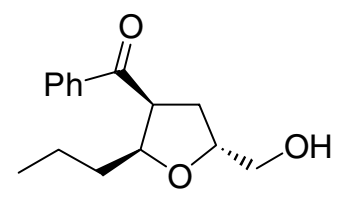

${ }^{1} \mathrm{H}$ NMR (400 MHz, $\left.\mathrm{CDCl}_{3}\right): \delta 7.97(2 \mathrm{H}, \mathrm{d}, J=8.3 \mathrm{~Hz}), 7.61-7.57(1 \mathrm{H}, \mathrm{m}), 7.49(2 \mathrm{H}, \mathrm{t}, J=$ $7.7 \mathrm{~Hz}), 4.49-4.44(1 \mathrm{H}, \mathrm{m}), 4.41-4.35(1 \mathrm{H}, \mathrm{m}), 4.22-4.16(1 \mathrm{H}, \mathrm{q}, J=7.5 \mathrm{~Hz}), 3.77-3.73(1 \mathrm{H}$, dd, $J=11.5,2.9 \mathrm{~Hz}), 3.59-3.55(1 \mathrm{H}, \mathrm{dd}, J=11.5,5.5 \mathrm{~Hz}), 2.68-2.61(1 \mathrm{H}, \mathrm{m}), 1.97(1 \mathrm{H}$, broad s), 1.95-1.88 (1H, ddd, $J=13.0,8.3,5.4 \mathrm{~Hz}), 1.49-1.15(3 \mathrm{H}, \mathrm{m}), 1.01-0.93(1 \mathrm{H}, \mathrm{m})$, $0.78(3 \mathrm{H}, \mathrm{t}, J=7.2 \mathrm{~Hz}) .{ }^{13} \mathrm{C} \mathrm{NMR}\left(100 \mathrm{MHz}, \mathrm{CDCl}_{3}\right): \delta 198.5,137.4,133.3,128.8,128.2$, 
80.8, 77.7, 65.3, 49.9, 33.1, 29.4, 19.4, 13.8. Anal. Calcd. for $\mathrm{C}_{15} \mathrm{H}_{20} \mathrm{O}_{3}: \mathrm{C}, 72.55 ; \mathrm{H}, 8.12$. Found C, 72.40; H, 7.96.

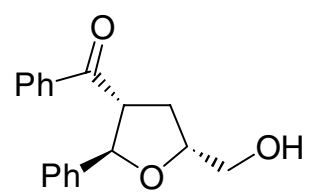

${ }^{1} \mathrm{H}$ NMR $\left(400 \mathrm{MHz}, \mathrm{CDCl}_{3}\right): \delta$ 7.80-7.78 $(2 \mathrm{H}, \mathrm{m}), 7.55-7.51(1 \mathrm{H}, \mathrm{m}), 7.41-7.23(7 \mathrm{H}, \mathrm{m})$, $5.42(1 \mathrm{H}, \mathrm{d}, J=7.8 \mathrm{~Hz}), 4.60-4.54(1 \mathrm{H}, \mathrm{m}), 4.10-4.03(1 \mathrm{H}, \mathrm{td}, J=9.8,8.2 \mathrm{~Hz}), 3.80-3.76$ $(1 \mathrm{H}, \mathrm{dd}, J=11.8,3.3 \mathrm{~Hz}), 3.74-3.70(1 \mathrm{H}, \mathrm{dd}, J=11.7,6.1 \mathrm{~Hz}), 2.54-2.47$ (1H, ddd, $J=12.6$, 8.3, $6.7 \mathrm{~Hz}), 2.25-2.17(1 \mathrm{H}$, ddd, $J=12.6,9.8,8.2 \mathrm{~Hz}) \cdot{ }^{13} \mathrm{C}$ NMR $\left(100 \mathrm{MHz}, \mathrm{CDCl}_{3}\right): \delta$ 199.1, 141.1, 137.0, 133.4, 128.63, 128.56, 128.4, 127.9, 125.8, 82.7, 80.3, 64.7, 55.5, 33.8. Anal. Calcd. for $\mathrm{C}_{18} \mathrm{H}_{18} \mathrm{O}_{3}$ : C, 76.57; H, 6.43. Found C, 76.42; H, 6.31.

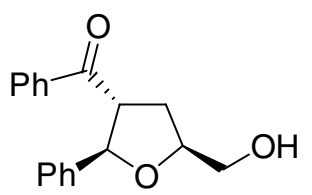

${ }^{1} \mathrm{H}$ NMR $\left(400 \mathrm{MHz}, \mathrm{CDCl}_{3}\right): \delta$ 7.81-7.78 $(2 \mathrm{H}, \mathrm{m}), 7.55-7.50(1 \mathrm{H}, \mathrm{m}), 7.44-7.24(7 \mathrm{H}, \mathrm{m})$, $5.30(1 \mathrm{H}, \mathrm{d}, J=7.6 \mathrm{~Hz}), 4.38-4.32(1 \mathrm{H}, \mathrm{m}), 4.07-3.92(2 \mathrm{H}, \mathrm{m}), 3.74-3.70(1 \mathrm{H}, \mathrm{dd}, J=11.8$, $4.8 \mathrm{~Hz}), 2.44-2.29(2 \mathrm{H}, \mathrm{m}) .{ }^{13} \mathrm{C} \mathrm{NMR}\left(100 \mathrm{MHz}, \mathrm{CDCl}_{3}\right): \delta$ 199.7, 140.6, 136.4, 133.4, 128.61, 128.56, 128.5, 128.1, 126.2, 83.7, 79.4, 64.5, 54.7, 33.4. Anal. Calcd. for $\mathrm{C}_{18} \mathrm{H}_{18} \mathrm{O}_{3}$ : C, 76.57; H, 6.43. Found C, 76.45; H, 6.33.

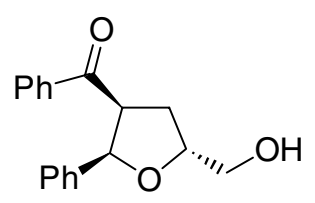

Characteristic ${ }^{1} \mathrm{H}$ signals: $\delta 5.47(1 \mathrm{H}, \mathrm{d}, J=7.3 \mathrm{~Hz}), 4.86-4.80(1 \mathrm{H}, \mathrm{m}), 3.85-3.81(1 \mathrm{H}, \mathrm{dd}, J$ $=11.7,3.2 \mathrm{~Hz}), 3.69-3.65(1 \mathrm{H}, \mathrm{dd}, J=11.6,5.8 \mathrm{~Hz}), 2.71-2.65(1 \mathrm{H}, \mathrm{ddd}, J=13.0,7.8,4.9$ $\mathrm{Hz}), 2.13-2.06$ (1H, ddd, $J=13.0,7.1,6.1 \mathrm{~Hz})$.

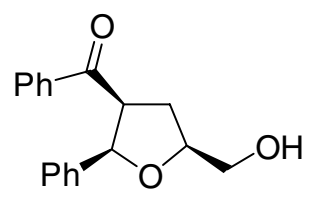


Characteristic ${ }^{1} \mathrm{H}$ signals: $\delta 5.29(1 \mathrm{H}, \mathrm{d}, J=8.1 \mathrm{~Hz}), 4.36-4.30(1 \mathrm{H}, \mathrm{m}), 4.10-4.07(1 \mathrm{H}, \mathrm{dd}, J$ $=11.9,2.5 \mathrm{~Hz}), 3.94-3.90(1 \mathrm{H}, \mathrm{dd}, J=11.7,4.9 \mathrm{~Hz}), 2.64-2.57(1 \mathrm{H}, \mathrm{ddd}, J=13.0,8.6,6.1$ $\mathrm{Hz}), 2.29-2.22(1 \mathrm{H}, \mathrm{td}, J=13.0,7.7 \mathrm{~Hz})$.

${ }^{13} \mathrm{C}$ NMR $\left(100 \mathrm{MHz}, \mathrm{CDCl}_{3}\right)$ of the mixture: $\delta$ 200.4, 199.1, 138.3, 137.5, 132.7, 132.6, 128.2, 128.12, 128.07, 128.04, 127.9, 127.8, 127.7, 126.8, 126.4, 83.5, 83.3, 80.2, 79.6, 65.3, $64.3,51.5,51.1,30.5,30.1$.

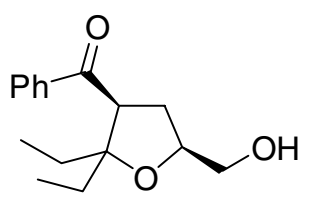

${ }^{1} \mathrm{H}$ NMR (400 MHz, $\left.\mathrm{CDCl}_{3}\right): \delta$ 7.92-7.90 (2H, m), 7.59-7.57 (1H, m), 7.50-46 (2H, m), 4.25$4.19(1 \mathrm{H}, \mathrm{m}), 4.10(1 \mathrm{H}, \mathrm{t}, J=7.7 \mathrm{~Hz}), 3.88-3.85(1 \mathrm{H}, \mathrm{dd}, J=11.6,2.8 \mathrm{~Hz}), 3.70-3.66(1 \mathrm{H}$, $\mathrm{dd}, J=11.5,4.9 \mathrm{~Hz}), 2.46-2.39(1 \mathrm{H}, \mathrm{m}), 2.20-2.13(1 \mathrm{H}, \mathrm{td}, J=12.9,7.4 \mathrm{~Hz}), 1.80-1.71(1 \mathrm{H}$, sextet, $J=7.3 \mathrm{~Hz}), 1.72(1 \mathrm{H}$, broad s), 1.59-1.46 $(2 \mathrm{H}, \mathrm{m}), 1.35-1.26(1 \mathrm{H}$, sextet, $J=7.3 \mathrm{~Hz})$, $1.00(3 \mathrm{H}, \mathrm{t}, J=7.3 \mathrm{~Hz}), 0.73(3 \mathrm{H}, \mathrm{t}, J=7.3 \mathrm{~Hz}) .{ }^{13} \mathrm{C} \mathrm{NMR}\left(100 \mathrm{MHz}, \mathrm{CDCl}_{3}\right): \delta 201.4$, 138.2, 133.2, 128.7, 128.2, 88.1, 77.9, 65.0, 50.9, 31.5, 28.4, 27.9, 8.24, 8.18. Anal. Calcd. for $\mathrm{C}_{16} \mathrm{H}_{22} \mathrm{O}_{3}$ : C, 73.25; H, 8.45. Found $\mathrm{C}, 73.15 ; \mathrm{H}, 8.33$.

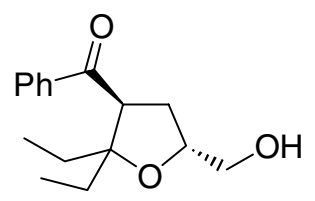

${ }^{1} \mathrm{H}$ NMR (400 MHz, $\left.\mathrm{CDCl}_{3}\right): \delta$ 7.90-7.88 $(2 \mathrm{H}, \mathrm{m}), 7.58-7.55(1 \mathrm{H}, \mathrm{m}), 7.49-7.45(2 \mathrm{H}, \mathrm{m})$, 4.33-4.28 (1H, m), 4.07-4.03 (1H, dd, $J=9.0,6.8 \mathrm{~Hz}), 3.80-3.77(1 \mathrm{H}, \mathrm{dd}, J=11.5,3.2 \mathrm{~Hz})$, 3.58-3.54 (1H, dd, $J=11.5,5.0 \mathrm{~Hz}), 2.62-2.55(1 \mathrm{H}, \mathrm{td}, J=12.8,7.3 \mathrm{~Hz}), 2.08(1 \mathrm{H}$, broad s), 2.04-1.97 (1H, ddd, $J=12.7,8.8,6.8 \mathrm{~Hz}), 1.82-1.73(1 \mathrm{H}$, sextet, $J=7.3 \mathrm{~Hz}), 1.71-1.62(1 \mathrm{H}$, sextet, $J=7.3 \mathrm{~Hz}), 1.51-1.42(1 \mathrm{H}$, sextet, $J=7.3 \mathrm{~Hz}), 1.23-1.14(1 \mathrm{H}$, sextet, $J=7.3 \mathrm{~Hz}), 0.97$ $(3 \mathrm{H}, \mathrm{t}, J=7.3 \mathrm{~Hz}), 0.77(3 \mathrm{H}, \mathrm{t}, J=7.3 \mathrm{~Hz}) .{ }^{13} \mathrm{C} \mathrm{NMR}\left(100 \mathrm{MHz}, \mathrm{CDCl}_{3}\right): \delta 200.5,138.3$, 133.0, 128.7, 128.0, 87.8, 77.1, 65.2, 51.2, 31.5, 29.0, 26.7, 8.1, 7.7. Anal. Calcd. for $\mathrm{C}_{16} \mathrm{H}_{22} \mathrm{O}_{3}$ : C, 73.25; H, 8.45. Found C, 73.11; H, 8.30. 


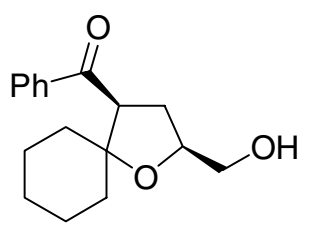

${ }^{1} \mathrm{H}$ NMR (400 MHz, $\left.\mathrm{CDCl}_{3}\right): \delta$ 7.95-7.93 $(2 \mathrm{H}, \mathrm{m}), 7.60-7.57(1 \mathrm{H}, \mathrm{m}), 7.51-7.47(2 \mathrm{H}, \mathrm{m})$, 4.25-4.19 (1H, m), 3.97-3.93 (1H, dd, $J=7.8,7.1 \mathrm{~Hz}), 3.91-3.88(1 \mathrm{H}, \mathrm{dd}, J=11.9,2.9 \mathrm{~Hz})$, 3.74-3.70 (1H, dd, $J=11.5,4.9 \mathrm{~Hz}), 2.47-2.40$ (1H ddd, $J=12.9,8.5,6.8 \mathrm{~Hz}$ ), 2.20-2.13 $(1 \mathrm{H}, \mathrm{td}, J=12.9,7.5 \mathrm{~Hz}), 1.92-1.87(1 \mathrm{H}, \mathrm{m}), 1.69-1.26(6 \mathrm{H}, \mathrm{m}), 1.10-1.02(2 \mathrm{H}, \mathrm{m}), 0.90-$ $0.82(1 \mathrm{H}, \mathrm{m}) .{ }^{13} \mathrm{C} \mathrm{NMR}\left(100 \mathrm{MHz}, \mathrm{CDCl}_{3}\right): \delta 201.1,137.9,133.3,128.7,128.4,84.5,76.9$, 64.9, 53.9, 36.7, 34.2, 30.8, 25.3, 22.7, 22.4. Anal. Calcd. for $\mathrm{C}_{17} \mathrm{H}_{22} \mathrm{O}_{3}$ : C, 74.42; $\mathrm{H}, 8.08$. Found C, 74.28; H, 7.97.

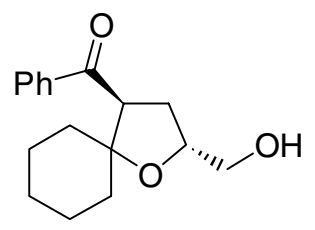

${ }^{1} \mathrm{H}$ NMR (400 MHz, $\left.\mathrm{CDCl}_{3}\right): \delta$ 7.95-7.93 $(2 \mathrm{H}, \mathrm{m}), 7.59-7.57(1 \mathrm{H}, \mathrm{m}), 7.50-7.46(2 \mathrm{H}, \mathrm{m})$, 4.38-4.32 (1H, m), 3.92-3.88 (1H, dd, $J=8.6,7.0 \mathrm{~Hz}), 3.80-3.76(1 \mathrm{H}, \mathrm{dd}, J=11.5,3.2 \mathrm{~Hz})$, 3.57-3.53 (1H, dd, $J=11.5,4.9 \mathrm{~Hz}), 2.65-2.58(1 \mathrm{H}, \mathrm{m}), 2.20(1 \mathrm{H}$, broad s), 2.04-1.97 $(1 \mathrm{H}$, ddd, $J=12.7,8.8,6.6 \mathrm{~Hz}), 1.80-1.38(8 \mathrm{H}, \mathrm{m}), 1.04-0.88(2 \mathrm{H}, \mathrm{m}) .{ }^{13} \mathrm{C}$ NMR $(100 \mathrm{MHz}$, $\left.\mathrm{CDCl}_{3}\right): \delta 199.8,137.9,133.1,128.6,128.4,84.4,77.0,65.2,54.8,38.8,31.8,30.9,25.3$, 23.1, 21.9. Anal. Calcd. for $\mathrm{C}_{17} \mathrm{H}_{22} \mathrm{O}_{3}$ : C, 74.42; H, 8.08. Found C, 74.36; H, 7.99. 